\title{
SPATIAL ANALYSIS OF BRAIN AND OTHER CNS CANCERS INCIDENCE IN IRAQ DURING 2000-2015
}

\author{
Muzahem Mohammed AL-Hashimi ${ }^{1}$ and Ahmed Naziyah Alkhateeb ${ }^{2}$ \\ ${ }^{1}$ Department of Statistics and Informatics, College of Computer science \& Mathematics, University of Mosul, Mosul, \\ Iraq \\ 2Department of Operation Research and Intelligent Techniques, College of Computer science \& Mathematics, \\ University of Mosul, Mosul, Iraq
}

Corresponding author: Muzahem Mohammed AL-Hashimi

Email: muzahim_alhashime@uomosul.edu.iq OR muzahim63@gmail.com

\section{ABSTRACT}

Brain and other CNS cancers have evidenced increase in Iraq over the study period (2000-2015). Spatial variation of brain and CNS cancers in Iraq at the district level has not been explored. This study aimed to explore the spatial patterns of the Age-Standardized Incidence Rates (ASIRs) of brain and CNS cancers throughout Iraq (except Kurdish region) during 2000-2015 using spatial autocorrelation analyses. Data were obtained from the Iraqi Cancer Registry. The ASIRs were calculated according to geographical region (provinces and districts) for each period (2000-2004, 2005- 2009, and 2010-2015). spatial statistical tools were employed to evaluate hotspots, cold spots, spatial clustering and outliers for each period. Results showed a spatial correlation with hotspots, cold spots, and detecting spatial outliers. This study identified 7 districts as high-risk areas for brain and CNS cancers during 2010-2015, including Al-Sadir, Al-Kadhimiyah, Adhamia, Al-Karkh, Al-Rissafa, and Al-Madain districts in Baghdad province) and southern region (Abu-Al-Khaseeb district in Al-Basrah provinces, and we have evidenced an increase of brain and CNS cancers incidence rates during 2010-2015. The government efforts should focus on those regions, and the factors related to the spatial pattern of the brain and CNS cancers incidence in Iraq should be investigated.

Keywords: Brain and CNS cancers, Hotspots, Cold spots, Spatial outliers

\section{INTRODUCTION}

Although the brain and other CNS cancers are rare, they cause morbidity and mortality that is disproportionate to their incidence ${ }^{1,2}$. In 2016, there were 330000 incident cases of brain and CNS cancers and 227000 deaths globally ${ }^{3}$, with ASIR 4.63 per 100 000. The ASIR was high in East Asia, central and western Europe, but low in Oceania and central and eastern sub-Saharan Africa $^{3}$. The incidence was higher in men than in women $^{3}$. Several environmental risk factors of the brain and other CNS cancers have been studied, but few are well establishment ${ }^{4}$. The following environmental risk factors have been investigated in epidemiological studies for association with the brain and other CNS cancers: Pesticides, Fertilizers, Formaldehyde, Synthetic rubber, Vinyl chloride, and Petrochemicals and petroleum ${ }^{4}$.

In Iraq (except Kurdish region), there were 1160 newly diagnosed brain and CNS cancers cases in $2015^{5}$, where the incidence had increased over the study period, from $2.882 \%$ in 2000 to $5.53 \%$ in 2015. The Iraqi Cancer Registry (ICR) was established in 1974 through close cooperation of Ministry of Health and the Iraqi Cancer Society. It is responsible for collecting and combining detailed information about cancer patients from all provinces of Iraq. The ICR publishes an annual report of Iraq cancer statistics including new cases, mortality for the provinces and district levels.

Iraq is a developing country located in western Asia, bordered by Iran in the East, Turkey in the North, Syria in the west and Jordan in the southwest, Saudi Arabia in the South, and Kuwait in the southeast. It has a total area of $437,072 \mathrm{~km}^{2}$. Administratively, the country is divided into 18 provinces, 117 districts. Iraq's climate is subtropics with the mean temperature range from $5{ }^{\circ} \mathrm{C}$ to $10{ }^{\circ} \mathrm{C}$ in the coldest month of January and up to $30{ }^{\circ} \mathrm{C}$ in the hottest month of August. The estimated population of Iraq as on mid-year 2020, according to UN data, is $40,222,493$. Figure 1 shows the location of Iraq. The spatial patterns at the province-level or district-level have not been explored in Iraq. Explaining how districts with high or low ASIRs of brain and CNS cancers cluster spatially may support government efforts to reduce the burden of brain and CNS cancers in specific areas of Iraq.

This study is the first in Iraq to examine whether the ASIRs of brain and CNS cancers tend to clustering, to identify where hot/cold spots appear (groups of districts with extremely high or low of the ASIRs of brain and CNS cancers), and to identify spatial outliers (districts with high or low of the ASIRs of brain and CNS cancers 
surrounded by districts with dissimilar values), during 2000 to 2015 .

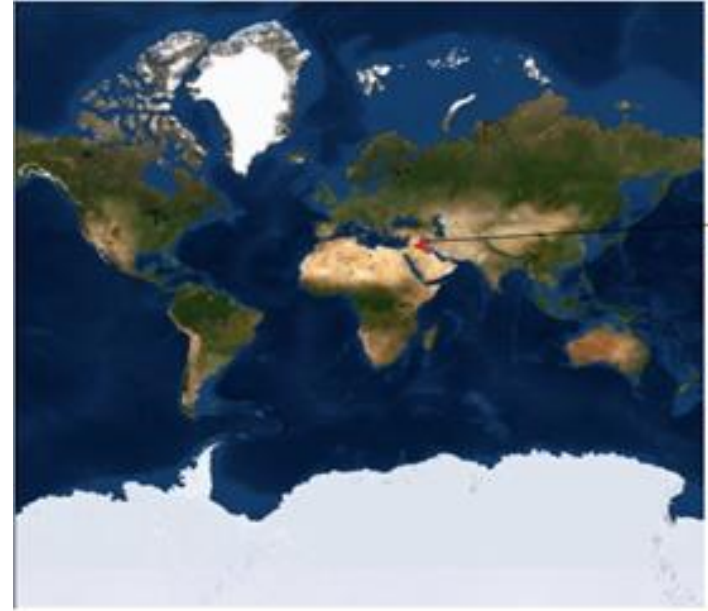

Figure 1: The location of Iraq

\section{METHODS}

\section{Data}

Brain and CNS cancers (ICD-10: C70-C72) records were obtained from the ICR in the Ministry of Health of Iraq. The ICR is responsible for aggregating data relating to every new cancer case in all provinces of Iraq ${ }^{5}$. The ICR annually published annual reports of cancer include detailed statistical data of cancer from all government and private hospitals throughout the country. In total, this study covers all provinces with the exception of three provinces in the Kurdish region (Erbil, Duhok and AlSulaymaniyah, for which the data is incomplete) with 83 districts in the country during 2000-2015. District level population data were obtained from the Central Organization for Statistics in the Ministry of Planning. This study examined brain and CNS cancer incidence data for 20002015. To enhance statistical stability, the data were divided into three periods (2000-2004, 2005-2009, and 2010-2015).

\section{Statistical methods}

The (ASIRs) were calculated according to geographical region (provinces and districts) for each time period (2000-2004, 2005- 2009, and 2010-2015) using the world standard population. The study employed the methods of spatial cluster, Global spatial autocorrelation, Local indicators of spatial association (Getis-Ord Gi* and Anselin Local Moran's I). The Global indexes of spatial autocorrelation - Moran's I was used to distinguish the spatial dependence across districts with respect to ASIRs. In other words, are districts with similar ASIRs of brain and CNS cancers located close together or are ASIRs of brain and CNS cancers randomly distributed across Iraq? Getis-Ord $\mathrm{Gi}^{*}$ and Anselin Local

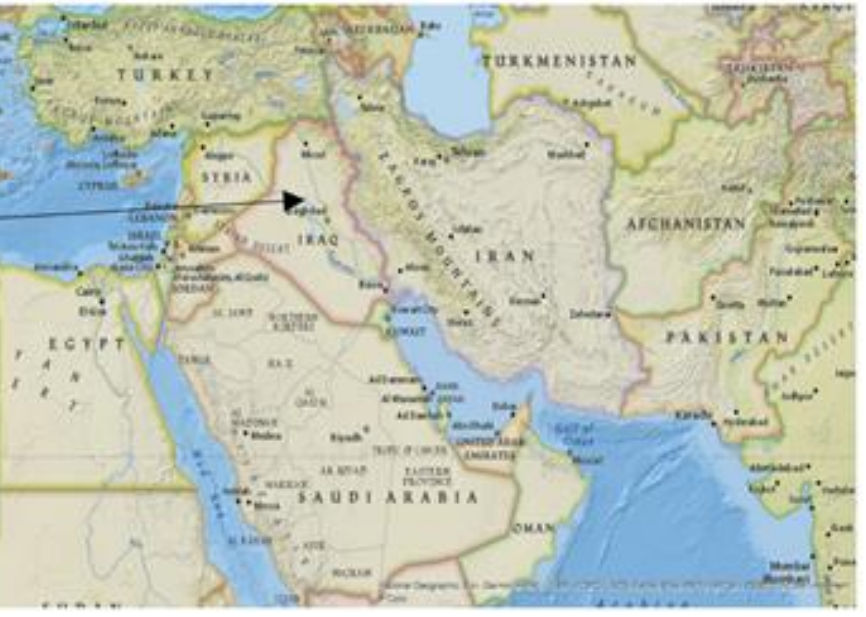

Moran's I were used to distinguish clusters of districts with high or low ASIRs and to distinguish spatial outliers. The descriptive analysis was performed using SAS (version 9.4) statistical software, and we used ArcMap 10.6 (Environmental Systems Research. Institute, Inc., Redlands, CA) for constructed maps of the brain and CNS cancers. Statistical significance was assumed at $\mathrm{p}<0.05$.

\section{Global spatial autocorrelation - Moran's I}

Global Moran's I was used to examine whether the pattern is clustered, dispersed, or random across districts with respect to ASIR. This statistic measures the spatial autocorrelation of districts in the provinces and the ASIRs simultaneously. The Global Moran's I statistic is given as follows ${ }^{6}$ :

Where:

$$
I=\frac{n \sum_{i} \sum_{j} W_{i, j}\left(X_{i}-\bar{X}\right)\left(X_{j}-\bar{X}\right)}{\sum_{i} \sum_{j} W_{i, j}\left(X_{i}-\bar{X}\right)^{2}}
$$

$X_{i}=$ the ASIRs of brain and CNS cancers for the ith district;

$X_{j}=$ the ASIRs of brain and CNS cancers for the jth district;

$\bar{X}=$ the mean of ASIRs of brain and CNS cancers for all of the districts in the study area;

$W_{i, j}=$ the spatial weight between pair of districts $i$ and $j$; and

$n=$ the total number of districts.

Moran's I scores usually ranges between -1.0 (indicates a dispersed pattern) and 1.0 (indicates a clustered pattern $)^{7}$.

\section{Getis-Ord $\mathrm{Gi}^{*}$ statistic}

The hot and cold spot analysis calculate the Getis-Ord $G_{i}^{*}$ tool for ASIRs in Iraq during 20002015. The Getis-Ord $G_{i}^{*}$ statistic is given as follows: 


$$
G_{i}^{*}=\frac{\sum_{j=1}^{n} W_{i, j} X_{j}-\bar{X} \sum_{j=1}^{n} W_{i, j}}{S \sqrt{\frac{n \sum_{j=1}^{n} W_{i, j}^{2}-\sum_{j=1}^{n} W_{i, j}}{n-1}}}
$$

Where:

$X_{i}=$ the ASIRs of brain and CNS cancers for the ith district;

$\bar{X}=$ the mean of ASIRs of brain and CNS cancers for all of the districts in the study area;

$W_{i, j}=$ the spatial weight between pair of districts $i$ and $j$;

$n=$ the total number of districts; and

$S=$ the standard deviation of the ASIRs of brain and CNS cancers in the study area, where $S=$ $\sqrt{\frac{\sum_{j=1}^{n} x_{j}^{2}}{n}-\bar{X}^{2}}$.

The $G_{i}^{*}$ statistic is a z-score, so no further calculations are required. The $G_{i}^{*}$ statistic returned for each feature in the dataset is a $Z$ score. The larger the $Z$ score, the more intense the clustering of high values. For statistically significant negative $Z$ scores, the smaller the $Z$ score is, the more intense the clustering of low values. The hotspot map can detect several hotspots and cold spots across Iraq.

The Anselin local Moran's I identify spatial outliers of districts with high or low ASIRs as well as spatial clusters. Clusters of districts with high ASIRs (HH = "high-high") are considered "hotspots", whereas clusters of districts with low ASIRs (LL = "low-low") are considered cold spots. Furthermore, Anselin local Moran's I identify districts with high ASIRs that are surrounded primarily by districts with low ASIRs (HL = "high(ow") and districts with low ASIRs that are surrounded primarily by districts with high ASIRs (LH = “low-high"). The Anselin local Moran's I statistic is given as follows:

$$
I_{i}=\frac{\left(X_{i}-\bar{X}\right) \sum_{j} W_{i, j}\left(X_{j}-\bar{X}\right)}{S^{2}}
$$

Where:

$X_{i}=$ the ASIRs of brain and CNS cancers for the ith district;

$\bar{X}=$ the mean of ASIRs of brain and CNS cancers for all of the districts in the study area;

$X_{j}=$ the ASIRs of brain and CNS cancers for the jth district;

$W_{i, j}=$ the spatial weight between pair of districts $i$ and $j$;

$n=$ the total number of districts; and

$S=$ the standard deviation of the ASIRs of brain and CNS cancer in the study area, where $S=$ $\sqrt{\frac{\sum_{j=1}^{n} x_{j}^{2}}{n}-\bar{X}^{2}}$.

\section{RESULTS}

During the period 2000-2015, a total of 13725 brain and CNS cancers incidences occurred in Iraq (except Kurdish region), accounting for $5.53 \%$ of all malignant tumors. There was a total of $7452(54.29 \%)$ in males and $6273(45.70 \%)$ in females, ratio: 1.19. The average age of the patients was 37.0 years, and $63.42 \%$ were $0-49$ years of age. According to the world standard population, the ASIR was 5.051 per 100,000 people and 5.435 for male and 4.667 for female. Baghdad and Babil provinces had the highest ASIR (7.1568 and 5.6398 per 100,000 people, respectively) of brain and CNS cancers among the country's provinces. Salah al-Deen had the lowest ASIR (2.8884 per 100,000 people) over the study years. The ASIRs at the district level ranged from 0.629 to 8.855 per 100,000 people. The national ASIRs increased from 3.7712 in 2000-2004 to 4.8052 in 2005-2009 and to 6.1292 per 100,000 population in 2010-2015 (Figure 2).

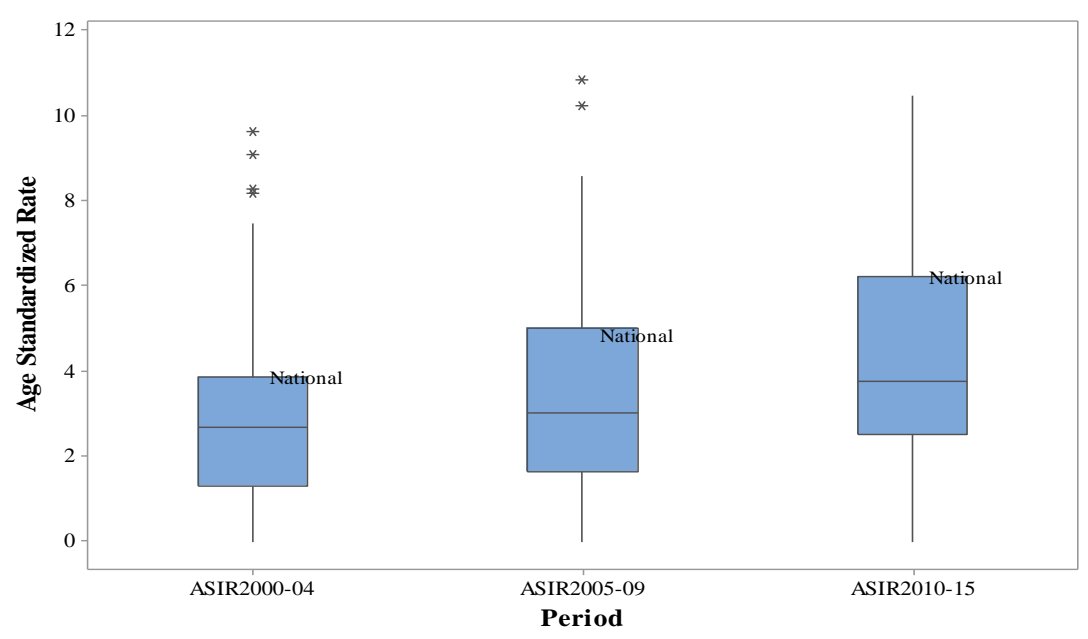

Figure 2: ASIRs of brain and CNS cancers over time 
Figures $3 \mathrm{~A}, 3 \mathrm{~B}$ and $3 \mathrm{C}$ displays the geographic distribution of the ASIRs of brain and CNS cancers during the periods 2000-2004, 2005-2009, and 2010-2015, respectively; the yellow scale represents the level of ASIRs and the browner the color, the higher the ASIRs.

Figure 3A shows that areas with the high ASIRs during the period 2000-2004 were concentrated in some districts in the northern, including $\mathrm{AL}$ Shikhan district in Nineveh province; some districts in the northeastern region, including ALHawija and Dakook districts in Kirkuk province; some districts in the north-central region, including Tikrit city in Salah al-Deen province; some districts in the central region, including AlRissafa district in Baghdad province. However, ASIRs in the southern and southwestern regions were relatively low.

Figure $3 \mathrm{~B}$ displays the distribution of ASIRs of brain and CNS cancers during the period 20052009. The areas with high ASIRs were located in the northern, central and southwestern regions; the highest ASIRs was in Kirkuk city in Kirkuk province, Al-Sadir and Al-Karkh districts in Baghdad province, and Al-Najaf city in Al-Najaf province. In 2010-2015, the areas with high ASIRs were focused in some districts in the central region, including most of the districts in Baghdad province, AL Sadir, AL Karkh, Al-Rissafa, AL Kadhimiyah and AL Adhamia districts; Al-Hilla city in Babil province; Karballa city in Karballa province; some districts of the southern region, including AL Basrah city and AL Zubair district in

Al-Basrah province, AL Nassiriyah city in Thi-Qar province; some districts of the southwestern region, including AL Najaf city in AL Najaf province; Kirkuk city in Kirkuk province in the northern region. However, ASIRs in the western regions were relatively low (Figure $3 \mathrm{C}$ ).

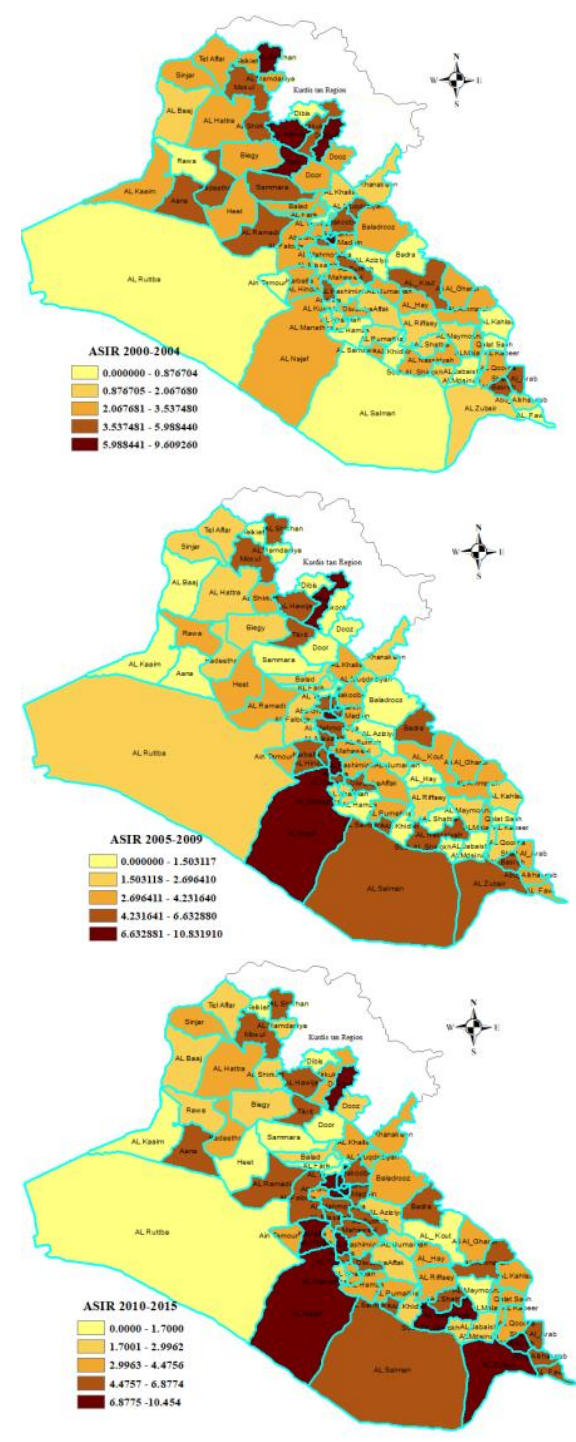

Figure 3: ASIRs (per 100,000) by districts. A show the ASIRs during, 2000 to 2004. B show the ASIRs during, 2005 to 2009. C show the ASIRs during, 2010 to 2015.

The global Moran's I test and the associated zscore for each of the three time periods 20002004, 2005-2009, and 2010-2015 demonstrated that there was significant spatial autocorrelation of district-level ASIRs of brain and CNS cancers. In other words, across Iraq, districts with similar ASIRs of the brain and CNS cancers exhibits spatial clustering for each of the three time periods 2000-2004, 2005-2009, and 2010-2015.

Significant spatial clusters of districts with hotspots and cold spots ASIRs of brain and CNS cancers, as assessed by the hotspot analysis (Getis-Ord $\mathrm{Gi}^{*}$ tool) for each of the three time periods 2000-2004, 2005-2009, and 2010-2015 can be seen in Figures $4 A, 4 B$ and $4 C$. In 20002004 (Figures 4A), 10 districts were identified as a hotspot, and 4 districts were identified as a cold spot. In 2005-2009 (Figures 4B), the number of districts designated as a hotspot declined to 9 
and the number of districts that designated as a cold spot decreased to 2 . In 2010-2015 (Figures 4C), the number of districts that appeared as a hotspot and cold spot remained the same as the period 2005-2009.

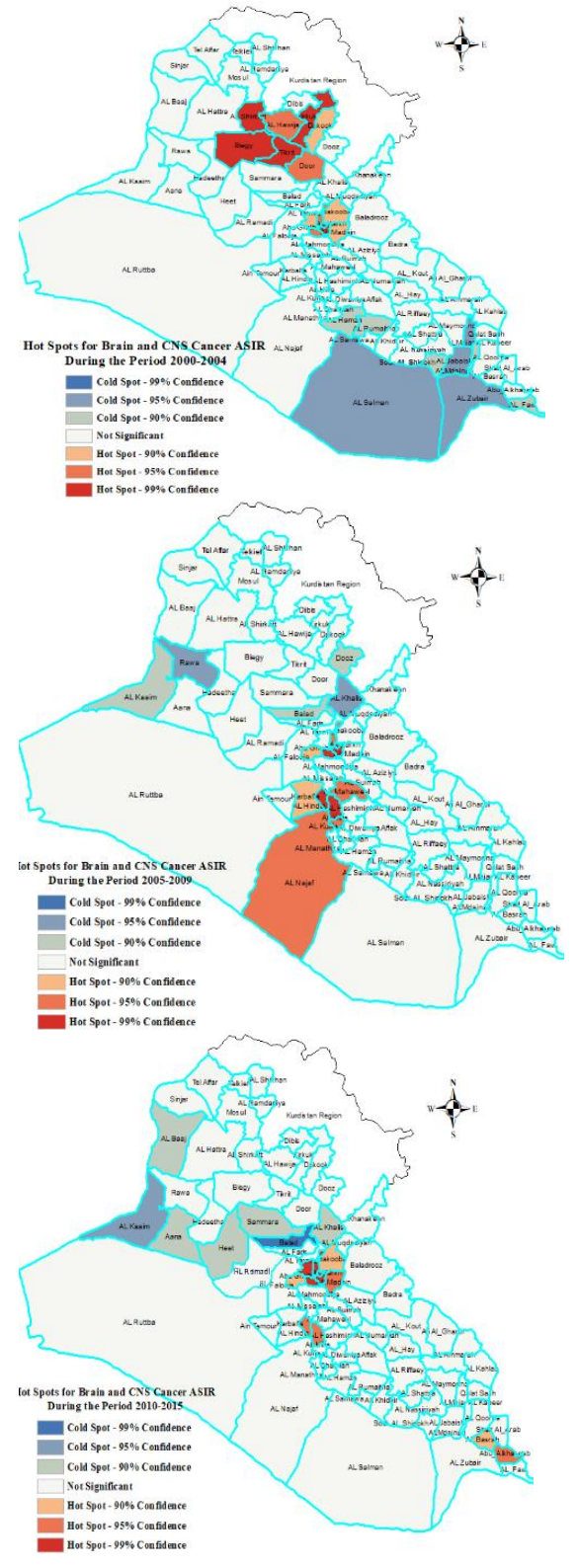

Figure 4: Hot and Cold Spots in ASIRs of brain and CNS cancers. A show the Hot and Cold Spots in ASIRs of brain and CNS cancers, 2000-2004. B show the Hot and Cold Spots in ASIRs of brain and CNS cancers, 2005-2009. C show the Hot and Cold Spots in ASIRs of brain and CNS cancers, 2010-2015.

A spatial cluster analysis (Anselin Local Moran's I) was used to examine clusters of districts with high or low ASIRs of brain and CNS cancers. In 2000-2004 (Figure 5A), the high-high districts were located in the provinces of Kirkuk (Kirkuk district), Salah al-Deen (Tikrit, Door, and AlShirkatt districts), and Baghdad (Al-Sadir, AlRissafa, Al-Adhamia, and Al-Madain districts).
Conversely, nine districts located in the provinces of Al-Diwaniya (Al-Diwaniya and Affak districts), Al-Muthanna (Al-Rumaitha and AlSalman districts), Thi-Qar (Sook Al-Shiookh and Al-jabaish districts), Maysan (Al-Majar Al-Kabeer district), and Al-Basrah (Al-Zubair and AlMdainah districts) showed low-low cluster patterns. Generally, these patterns changed in 2005-2009 (Figure 5B), the notable changes were in Kirkuk, Tikrit, Door, Al-Shirkatt, Al-Sadir, and Al-Madain, which no longer a hotspot.

In 2005-2009 (Figure 5B), The high-high appeared in the provinces of Baghdad (Al-Rissafa, AlAdhamia, and Al-Karkh districts), Babil (AlMahaweel and Al-Hilla districts), Karballa (AlHindia district), and Al-Najaf (Al-Najaf and AlKuffa). The cold spots districts were located in the provinces of Diyala (Al-Khalis district), Salah al-Deen (Dooz district), Al-Anbar (Rawa and AlKaaim districts). In 2010-2015 (Figure 5C), there were seven districts showed high-high cluster patterns, included Baghdad (Al-Sadir, AlKadhimiyah, Adhamia, Al-Karkh, Al-Rissafa, and Al-Madain districts), and southern region (Abu-AlKhaseeb district in Al-Basrah province).

The cold spots districts were located in the provinces of Al-Anbar (Heet district), Salah alDeen (Balad district), and Diyala (Al-Khalis district). Additionally, several districts were labelled as spatial outliers. In 2000-2004 (Figure $5 \mathrm{~A})$, there were three districts identified as the high-low type, where the ASIRs of brain and CNS cancers were high, but the district was surrounded by a cold spot, these districts were located in the provinces of Al-Najaf (Al-Najaf district), Al-Muthanna (Al-Samawa district), and Thi-Qar (Al-Nassiriyah district). Conversely, there were 3 districts identified as the low-high category, where the ASIRs of brain and CNS cancers were low, but the district was surrounded by a hotspot, these districts were located in the provinces Nineveh (Al-Hamdaniya district), Kirkuk (Dibis district), and Salah alDeen (Biegy district). In 2005-2009 (Figure 5B), we identified three districts spatial outliers, included one neighborhood with high ASIRs of brain and CNS cancers surrounded by a cluster of low ASIRs of brain and CNS cancers (high-low) (Heet district in Al-Anbar province); and two neighborhood with low ASIRs of brain and CNS cancers surrounded by a cluster of high ASIRs of brain and CNS cancers (low-high) (Dibis district in Kirkuk province and Al-Hamza district in AlDiwaniya province). 
During the period from 2010 to 2015 (Figure 5C), we identified five districts spatial outliers, included three neighborhood with high brain and CNS cancers ASIR surrounded by a cluster of low ASIRs of brain and CNS cancers (high-low) (Aana and Al-Ramadi districts in Al-Anbar province and Dakook district in Kirkuk province); and two neighborhood with low ASIRs of brain and CNS cancers surrounded by a cluster of high ASIRs of brain and CNS cancers (low-high) (Abu-Graib in Baghdad province and Al-Hindia district in Karballa province).

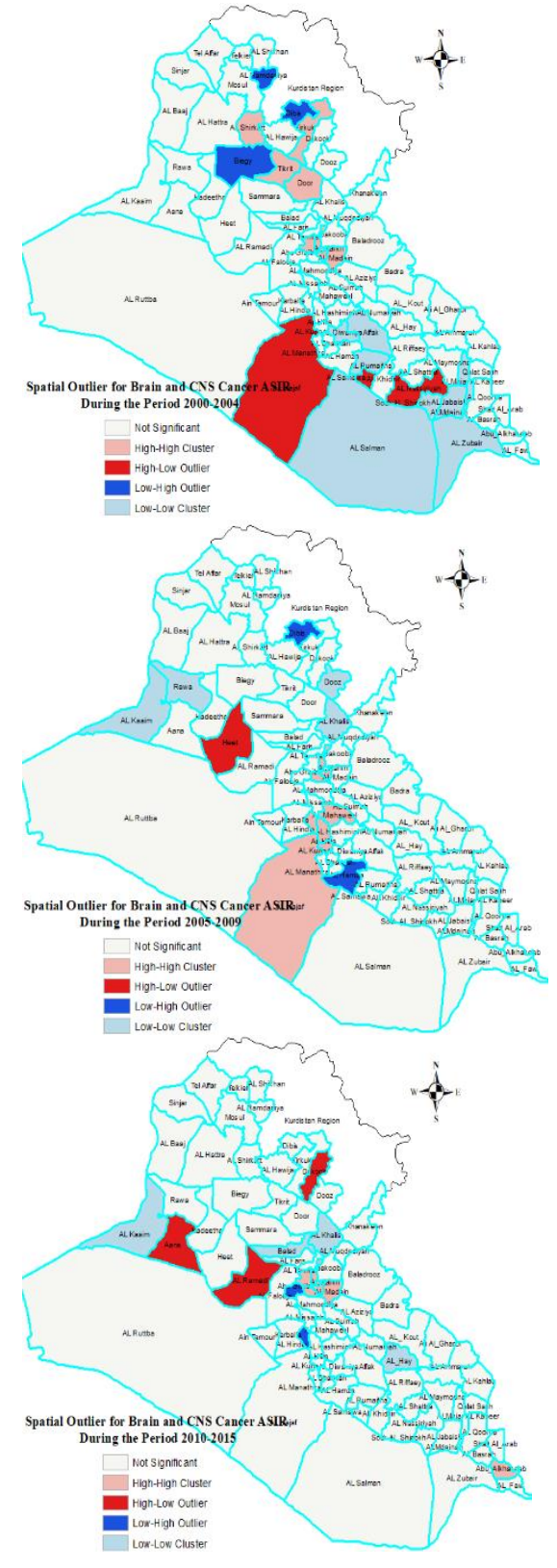

Figure 5: Clusters and spatial outliers in ASIRs of brain and CNS cancers. A show the Clusters and spatial outliers in ASIRs of brain and CNS cancers, 2000-2004. B show the Clusters and spatial outliers in ASIRs of brain and CNS cancers, 20052009. C show the Clusters and spatial outliers in ASIRs of brain and CNS cancers, 2010-2015.

\section{DISCUSSION}

Spatial variation of brain and CNS cancers in Iraq at the district level has not been explored. This study is the first to explore the geographic distribution of the brain and CNS cancers in Iraq using spatial autocorrelation analyses.

In this study, we found that brain and CNS cancers was the fifth most common type of cancer in Iraq, contributing $5.53 \%$ of the total number of new cases diagnosed in 2000 to 2015, with an ASIR of 5.051 per 100,000, which is lower than the ASIR in Albania, Sweden, Armenia, Serbia, Macedonia ${ }^{8}$, Nordic countries ${ }^{3}$, and $\operatorname{Iran}^{9}$ and higher than global $\mathrm{ASIR}^{3,8}{ }^{8}$, Korea ${ }^{10}$, the Gulf Cooperation Council (GCC) countries ${ }^{11}$, Saudi Arabia ${ }^{12,13}$. We found that ASIR of brain and CNS cancers higher in males than females, which is in line with most studies ${ }^{8-10}, 14,15$. Our results suggested that ASIRs of brain and other CNS cancers increased significantly during the period 2000 to 2015.

Worldwide, brain and other CNS cancers are rare, representing $1.6 \%$ of all cancers in both sexes ${ }^{16}$. The incidence of brain and other CNS cancers differs worldwide, East Asia was the region with the most incident cases of CNS cancer for both sexes in 2016, followed by western Europe, and south Asia. It is not known what causes brain and other CNS cancers ${ }^{4}, 17$. however, A variety of factors have been identified that may increase a person's chances of developing brain and other CNS cancers. Recent studies indicate that exposure to radiation, hormonal factors, nutrition, alcohol, smoking, Fertilizers, Air pollution, Electromagnetic fields, and family history may increase person's risk of developing brain and another CNS cancers ${ }^{4,6,14}$.

This study demonstrates geographic variation and risk clusters in ASIRs of brain and CNS cancers at the district level in Iraq. Results of global indicators of spatial autocorrelation confirm that ASIRs of brain and CNS cancers exhibit spatial autocorrelation. In other words, districts with high ASIRs of brain and CNS cancers tend to cluster together geographically. Conversely, districts with low ASIRs of brain and CNS cancers also tend to locate closer together. Using of Getis-Ord $\mathrm{Gi}^{*}$ tool of the spatial distribution, we were able to detect several hotspots and cold spots in Iraq that represent clusters of districts with significantly high or low ASIRs of brain and CNS cancers. 
In the present study, relatively high ASIRs of brain and CNS cancers were mainly focused in some districts in the northern and central regions (specifically most districts of Baghdad province were higher than those in other provinces of Iraq) in Iraq, while relatively low rates were concentrated in the southeastern and northwestern regions during the three study periods 2000-2004, 2005-2009, and 2010-2015.

The causes of global variation in incidence of brain and CNS cancers are unclear, but they may be partly explained by differences in risk factors such as a genetic susceptibility, environmental, lifestyle exposures ${ }^{18}$. However, it is beyond the aim of this paper to know the reasons of geographical variation of brain and other CNS cancers incidence in Iraq. The causes of this spatial pattern of brain and other CNS cancers in Iraq are unknown, but they may be due to exposure to lifelong, variations in socioeconomic status and health behaviors. However, the reasons for the higher incidence rates in some districts merits further investigation.

\section{CONCLUSIONS}

In summary, there is substantial geographic variation in ASIRs of brain and CNS cancers among Iraq. districts with high and low ASIRs of brain and CNS cancers tend to cluster together. Explaining how districts with high or low ASIRs of brain and CNS cancers cluster spatially may support government efforts to reduce the burden of brain and CNS cancers in specific areas of Iraq. Results of this study may be considered by public health practitioners as the cornerstone for setting brain and CNS cancers control plan and programs. Additionally, results may be used as a reference for the officials in the estimation of the need of labor force, medical and technical equipment and cancer medications toward highneed areas. Our finding determined clusters of areas with high brain and CNS cancers incidence in Iraq, specifically in the central (including AlSadir, Al-Kadhimiyah, Adhamia, Al-Karkh, AlRissafa, and Al-Madain districts in Baghdad province) and southern region (Abu-Al-Khaseeb district in Al-Basrah provinces). The government efforts should focus on those regions, and the factors related to spatial pattern of brain and CNS cancers incidence in Iraq should be investigated.

\section{Acknowledgments}

We would like to express our thanks to the staff of Iraqi Cancer Board-Ministry of Health for their effort for providing data access. We are grateful to university of Mosul, for supporting our work.

\section{Conflict of interest}

We declare that we have no conflicts of interest to disclose regarding this manuscript.

\section{Funding}

This manuscript received no specific grant from any funding agency in the public or commercial sectors.

\section{REFERENCES}

1. Leece $R, X u J$, Ostrom $Q T$, Chen $Y$, et al., Global incidence of malignant brain and other central nervous system tumors by histology, 20032007. Neuro-oncology 2017; 19(11):1553-64.

2. Gittleman HR, Ostrom QT, Rouse CD, et al., Trends in central nervous system tumor incidence relative to other common cancers in adults, adolescents, and children in the United States, 2000 to 2010. Cancer 2015; 121(1): 102-12.

3. Patel AP, Fisher JL, Nichols E, et al., Global, regional, and national burden of brain and other CNS cancer, 19902016: a systematic analysis for the Global Burden of Disease Study 2016. The Lancet Neurology 2019; 18(4):376-93.

4. McNeill K.A., Epidemiology of brain tumors. Neurologic clinics 2016; 34(4):981-98.

5. Annual Statistical Report 2015. Planning Directorate, Ministry of Health/ Environment, Republic of Iraq 2016: 4-5.

6. Waller LA. \& Gotway CA., Applied spatial statistics for public health data. John Wiley \& Sons, 2004: 227 234.

7. Legendre P. \& M.J. Fortin, Spatial pattern and ecological analysis. Vegetatio 1989; 80(2):107-38.

8. Khodamoradi F, Ghoncheh M, Pakzad $\mathrm{R}$, et al., The incidence and mortality of brain and central nervous system cancer and their relationship with human development index in the world. World Cancer Res J 2017; 4:e985. 
9. Araghi M, Roshandel G, HasanpourHeidari $S$, et al., Incidence of Malignant Brain and Central Nervous System Tumors in Golestan, Iran, 2004-2013. Archives of Iranian Medicine (AIM) 2020; 23(1)

10. Jung KW, Won $\mathrm{YJ}$, Kong $\mathrm{HJ}$, et al., Cancer statistics in Korea: incidence, mortality, survival, and prevalence in 2016. Cancer research and treatment: official journal of Korean Cancer Association 2019; 51(2):417.

11. Al-Madouj A, Eldali A, Al-Zahrani AS, et al., Ten-year cancer incidence among nationals of the GCC states 1998-2007. Gulf Center for Cancer Control and Prevention. 2011.

12. Council $\mathrm{S}$ health, Cancer Incidence Report. Saudi Arabia, Saudi Cancer Registry 2014: 53-54.

13. Almutrafi A, Bashawry $Y$, AlShakweer $W$, et al., The Epidemiology of Primary Central Nervous System Tumors at the National Neurologic Institute in Saudi Arabia: A Ten-Year Single-Institution Study. Journal of Cancer Epidemiology 2020;2020.

14. Piñeros $M$, Sierra MS, Izarzugaza MI, et al., Descriptive epidemiology of brain and central nervous system cancers in Central and South
America. Cancer epidemiology 2016; 44:S141-9.

15. Salhab HA, Khachfe HH, Fares MY, et al., Central nervous system cancers in the Middle East and North Africa (MENA) region: where does Lebanon stand. Chinese clinical oncology. 2020 Apr 23.

16. Bray F, Ferlay J, Soerjomataram I, et al., Global cancer statistics 2018: GLOBOCAN estimates of incidence and mortality worldwide for 36 cancers in 185 countries. CA: $a$ cancer journal for clinicians 2018; 68(6):394-424.

17. FARMANFARMA KK, Mohammadian M, Shahabinia Z, et al., BRAIN CANCER IN THE WORLD: AN EPIDEMIOLOGICAL REVIEW. World Cancer Research Journal 2019; 6:5.

18. Darefsky A.S. \& R. Dubrow, International variation in the incidence of adult primary malignant neoplasms of the brain and central nervous system. Cancer Causes \& Control 2009; 20(9):1593-604. 